\title{
Uso de fármacos psicoestimulantes en drogodependencias
}

\author{
Carlos Roncero; Miguel Casas
}

Servicio de Psiquiatría. Hospital Universitario Valle Hebrón. Barcelona

Universidad Autónoma de Barcelona

Enviar correspondencia a:

Dr. Carlos Roncero Alonso. Servicio de Psiquiatría. Escuela de Enfermería 5 planta. Hospital Universitario Valle Hebrón.

Paseo Vall d'Hebron. 119-129 Barcelona. 08025-Barcelona

croncero@vhebron.net. Teléfono: 9348942 95. Fax: 934894587

\section{RESUMEN}

El uso de medicamentos estimulantes es una cuestión de plena actualidad en psiquiatría, aunque su utilización y prescripción es controvertida. Fármacos como el metilfenidato, las anfetaminas, o el modafinilo están siendo utilizados y estudiados en distintas enfermedades psiquiátricas como el trastorno por déficit de atención e hiperactividad (TDAH), la dependencia de cocaína, en trastornos del sueño y en la depresión resistente. Todos estos fármacos tienen en común, igual que las drogas de abuso, que son medicamentos que actúan sobre el sistema dopaminérgico, que constituye la base neurobiológica del refuerzo fisiológico. Los estimulantes como el metilfenidato o el modafinilo son fármacos eficaces en el TDAH y han sido estudiados en el tratamiento de la dependencia de cocaína. En niños con TDAH el metilfenidato es un factor protector para el desarrollo de drogodependencias. Existen estudios experimentales con modafinilo que muestran la utilidad del fármaco en la dependencia de cocaína, aunque son estudios preliminares, por lo que no se debe considerar que este totalmente demostrado que los fármacos psicoestimulantes sean eficaces en el tratamiento de esta dependencia. Aunque no son conocidos todos los mecanismos fisiopatológicos, parece crítico que el refuerzo, y por lo tanto el riesgo de dependencia, aparece cuando se producen incrementos rápidos dopaminérgicos y que los efectos terapéuticos aparecen cuando son lentos y mantenidos. Las características de uso a dosis bajas administradas por vía oral disminuyen el riesgo de abuso. Para realizar una adecuada prescripción es necesario aclarar, definitivamente, los mecanismos neuroquímicos en los que intervienen, y sus indicaciones en drogodependencias.

Palabras clave: fármacos estimulantes, drogodependencias, metilfenidato, modafinilo.

\section{ABSTRACT}

Stimulant drugs prescription is a controversial and current topic in psychiatry. Drugs such as methylphenidate, amphetamine compounds and modafinil have been trialed and used in attention deficit hyperactivity disorder (ADHD), sleep conditions, cocaine dependence and as an adjunct to antidepressants for depression. All these drugs, like stimulant drugs abuse, increase extracellular dopamine in the brain. This effect is associated with reinforcing as well as therapeutic effects. Methylphenidate and modafinil treatment of ADHD are associated with a reduced risk for later substance abuse among ADHD patients. There is evidence of the beneficial effects of the use of modafinil in cocaine dependence, altough there isn't conclusive evidence for the stimulants' efficacy in treatment of the stimulants' dependence. At this time, the physiopathology of drug abuse and dependence is unknown, but it's known that the very critical point is that the reinforcing effects are associated with rapid changes in dopamine increases, whereas the therapeutic effects are associated with slowly and smoothly rising dopamine levels, such as are achieved with low doses and oral administration. Due to this, it's necessary to study the neurobiological bases on which stimulants drugs are related, and their clinical use in dependence treatments.

Key words: stymulants drugs, drug dependence, methylphenidate, modafinil
$\mathbf{E}$ uso de medicamentos estimulantes es una cuestión de plena actualidad en psiquiatría su utilización y prescripción es controvertida, en ocasiones sin que existan dudas basadas en los conocimientos científicos actuales. Fármacos como el metilfenidato, las anfetaminas, o el modafinilo están siendo utilizados y estudiados en distintas enfermedades psiquiátricas como son el trastorno por déficit de atención e hiperactividad (TDAH), el tratamiento de la dependencia de cocaína, en trastornos del sueño y como potenciadores en el tratamiento de la depresión resistente. Todos estos fármacos tienen en 
común que son medicamentos prodopaminérgicos ya que inhiben su recaptación o aumentan su recambio, potenciando las acciones de la dopamina, al aumentar su concentración extracelular. (Volkow y Swanson, 2003). Es conocido que el sistema dopaminérgico constituye la base neurobiológica del refuerzo fisiológico y que las drogas de abuso, como la cocaína o las sustancias anfetamínicas actúan sobre este sistema. Sin embargo y a pesar de que el consumo de estimulantes, como el metilfenidato, puede producir efectos conductuales asociados con el abuso de sustancias y se debe ser consciente del riesgo de abuso, es relevante conocer que sus propiedades farmacocinéticas reducen su riesgo (Kollins, 2003).

El metilfenidato es uno de los fármacos emergentes en el panorama médico actual, ha sido estudiado ampliamente en el TDHA, tanto en su presentación de liberación inmediata como retardada, demostrando su utilidad en su tratamiento, tanto en niños y niñas (Biederman, Wigal, Spencer, McGough y Mays, 2006a) como en adultos a dosis de $1 \mathrm{mg} / \mathrm{kg} /$ día o incluso más (Biederman, Mick, Spencer, Surman, Hammerness, Doyle et al. 2006b), reduciendo significativamente los síntomas de inatención e hiperactivitividad/impulsividad (Biederman, Mick, Surman, Doyle, Hammerness, Harpold, et al., 2006c). Otro fármaco novedoso en nuestro medio, el modafinilo, también esta siendo estudiado en el TDAH, existiendo evidencias de su utilidad (Ballon y Feifel, 2006). Además se ha utilizado en algunos trastornos del sueño, sedación postanestésica, tratamiento de la dependencia y su abstinencia y como coadyuvante con los antidepresivos (Ballon y Feifel, 2006).

Sin embargo el uso de estos fármacos en el tratamiento de conductas adictivas es controvertido. En pro de su utilización se puede argumentar que pueden evitar, en algunos pacientes el desarrollo de adicción. Se conoce que la presencia de trastornos psiquiátricos en la edad infanto-juvenil, se relaciona con el desarrollo de drogodependencias en la vida adulta. Aunque se podría presuponer que el contacto con fármacos estimulantes a estas edades produciría un mayor riesgo de abusar o presentar dependencia de sustancias, su uso ha relacionado con mejor evolución en niños y adolescentes con TDAH e incluso es un factor protector. En este sentido el tratamiento con metilfenidato se ha asociado con la disminución del riesgo de aparición de drogodependencias en la vida adulta, cuando se compara con niños enfermos de TDAH que no han sido tratados (Katusic, Barbaresi, Colligan, Weaver, Leibson y Jacobsen, 2005), por lo que la prescripción de estos fármacos, paradójicamente, son un factor protector para el desarrollo de adicción. En otro sentido, aunque existen estudios experimentales con modafinilo que muestran la utilidad del fármaco en la dependencia de cocaína, en la actualidad no se puede afirmar que estos fármacos hallan demostra- do definitivamente su utilidad en el tratamiento de la dependencia de estimulantes u otras drogas. Además, dados los efectos fisiológicos cardiacos que los fármacos estimulantes producen se debe tener precaución y ser cauto cuando se realiza tratamientos con estos fármacos y evaluar su función cardiaca. Cuando se ha estudiado en adultos los medicamentos estimulantes pueden producir un aumento menor, pero estadísticamente significativo, de la tensión arterial sistólica, diastólica y la frecuencia cardiaca (Biderman, Mick, Surman, Doyle, Hammerness, Harpold et al. 2006c; Wilens, Hammerness, Biederman, Kwon, Spencer, Clark et al., 2006). Dichos cambios se ha detectado con medicaciones tanto estimulantes (metilfenidato, compuestos anfetamínicos, pemolina), como otros fármacos utilizados en el tratamiento de trastornos adictivos, pero no clasificados como estimulantes (bupropion, desipramina) debido a sus efectos catecolinérgicas, ya que presentan efectos cronotrópicos, que se deben evaluar al comienzo del tratamiento y periódicamente (Wilens, Hammerness, Biderman, Kwon, Spencer, Clark, et al., 2006). Por lo que es recomendable una monitorización básica de estos parámetros en pacientes que reciben el tratamiento. Aunque esto no debe ser un óbice para el manejo de estos fármacos, si existe una adecuada indicación, ya que una evaluación médica básica debería hacerse en todos los pacientes que reciben psicofármacos.

En la actualidad los clínicos necesitan poder discernir cuales son los factores asociados al abuso o dependencia de estas sustancias o a sus efectos médicos en el tratamiento de las enfermedades psiquiátricas, y su posible uso en el abordaje la dependencia de sustancias tanto en los programas de desintoxicación como, incluso, de mantenimiento. Para ello es muy importante discriminar y conocer los efectos y las variables clínicas que pueden influir tanto en las propiedades reforzantes, y por tanto de abuso como en las terapéuticas para potenciar estas y evitar los riesgos de adicción y mal uso. Volkow y Swanson, (2003) han identificado cuatro posibles variables que incluyen la dosis administrada del fármaco, aspectos farmacocinéticos, como es la vía de administración, las diferencias individuales que pueden condicionar los niveles necesarios para que aparezcan los efectos reforzantes, ligados al abuso o los terapéuticos, relacionados con la disminución de síntomas. Finalmente otro aspecto clave es el contexto, ya que los efectos son modulados por los lugares donde se consumen y los distintos rituales de autoadministration y condicionamiento, muy diferentes del uso clínico, pautado por profesionales, con demandas externas de baja actividad y focalizados en el control de síntomas. En relación a las propiedades farmacocinéticas se conoce que los efectos reforzantes están asociados a incrementos rápidos en las concentración del fármaco, y por lo tanto, a incrementos súbitos dopaminérgicos y que dichos efectos aparecen más relacionados con la 
administración intravenosa o inhalada. Por otra parte los efectos terapéuticos están relacionados con el aumento progresivo en la concentración y presumiblemente con el incremento gradual y suave, relacionados con el tratamiento oral.

Aunque no son conocidos todo los mecanismos fisiopatológicos, en la actualidad, parece crítico que el refuerzo aparece cuando se elicitan rápidos e importantes incrementos dopaminérgicos y los efectos terapéuticos aparecen cuando son lentos y mantenidos. Estas características de uso clínico, dosis bajas administradas por vía oral y con un incremento progresivo disminuyen el riesgo de abuso (Volkow y Swanson, 2003), hecho que se ve favorecido, en el caso del metilfenidato, por sus propiedades farmacocinéticas que reducen este riesgo (Kollins, 2003).

El futuro del tratamiento con psicoestimulantes en drogodependencias parece prometedor aunque, para evitar falsas controversias, es fundamental que se realicen más estudios sistemáticos que aclararen definitivamente los mecanismos neuroquímicos en los que intervienen estos fármacos, sus indicaciones precisas y utilidad en el tratamiento de las drogodependencias.

\section{REFERENCIAS}

Ballon JS, Feifel D. A systematic review of modafinil: Potential clinical uses and mechanisms of action. J Clin Psychiatry. 2006; 67: 554-66.
Biederman J, Wigal SB, Spencer TJ, McGough JJ, Mays DA. A post hoc subgroup analysis of an 18-day randomized controlled trial comparing the tolerability and efficacy of mixed amphetamine salts extended release and atomoxetine in school-age girls with attention-deficit/ hyperactivity disorder. Clin Ther. 2006a; 28: 280-93.

Biederman J, Mick E, Spencer T, Surman C, Hammerness $P$, Doyle R, et al. An open-label trial of OROS methylphenidate in adults with late-onset ADHD. CNS Spectr. 2006b; 11: 390-6.

Biederman J, Mick E, Surman C, Doyle R, Hammerness P, Harpold $T$, et al. A randomized, placebo-controlled trial of OROS methylphenidate in adults with attentiondeficit/hyperactivity disorder. Biol Psychiatry. 2006c 1; 59: 829-35.

Katusic SK, Barbaresi WJ, Colligan RC, Weaver AL, Leibson CL, Jacobsen SJ. Psychostimulant treatment and risk for substance abuse among young adults with a history of attention-deficit/hyperactivity disorder: a population-based, birth cohort study. J Child Adolesc Psychopharmacol. 2005; 15: 764-76.

Kollins SH. Comparing the abuse potential of methylphenidate versus other stimulants: a review of available evidence and relevance to the ADHD patient. J Clin Psychiatry. 2003; 64 Suppl 11: 14-8.

Volkow ND, Swanson JM. Variables that affect the clinical use and abuse of methylphenidate in the treatment of ADHD. Am J Psychiatry. 2003; 160: 1909-18.

Wilens TE, Hammerness PG, Biederman J, Kwon A, Spencer TJ, Clark S, et al. Blood pressure changes associated with medication treatment of adults with attention-deficit/hyperactivity disorder. J Clin Psychiatry. 2005; 66: 253-9. 
